Sympozjum

Rok XXIV 2020, nr 2(39), s. 83-123

\author{
ks. Leszek Poleszak SCJ \\ Wyższe Seminarium Misyjne Księży Sercanów w Stadnikach \\ ORCID: 0000-0001-5408-0809; e-mail: Ipoleszak@scj.pl \\ https://doi.org/10.4467/25443283SYM.20.022.12953
}

\title{
SORGENTI CRISTOLOGICHE DELLA VITA CONSACRATA
}

ŹRÓDŁA CHRYSTOLOGICZNE ŻYCIA KONSEKROWANEGO

CHRISTOLOGICAL SOURCES OF THE CONSECRATED LIFE

\begin{abstract}
Abstrakt
Artykuł ma za zadanie wskazać na chrystocentryczne źródła życia konsekrowanego. Jezus Chrystus jest dla osób konsekrowanych założycielem i wzorem obranej przez nich formy życia. Syn Boży jawi się jako wzór konsekracji opartej na profesji rad ewangelicznych, która w sensie ścisłym oznacza naśladowanie formy życia praktykowanej przez Niego. Artykuł wskazuje także na paschalny wymiar konsekracji, która zakorzeniona jest w konsekracji chrzcielnej, będącej początkiem życia Bożego w człowieku, oraz w Eucharystii. Naśladując Chrystusa czystego, ubogiego i posłusznego, osoby konsekrowane stają się świadkami Jego obecności w świecie.

Słowa kluczowe: Jezus Chrystus, życie konsekrowane, konsekracja, świadectwo, świętość, powołanie, wiara, Kościół
\end{abstract}




\begin{abstract}
The goal of the article is to present the Christocentric sources of the consecrated life. Jesus Christ is for all the consecrated people their founder and pattern for the chosen by them form of life. The son of God is indeed the model of consecration based on the profession of the evangelical counsels which in the strict sense means to follow the form of life He has chosen and practised himself. The article picked out on the paschal dimension of the consecration, which is deeply rooted in the baptismal consecration, which is the beginning of the God's life in human as well as in the Eucharist. Following Christ who is chaste, poor and obedient the consecrated people become the witnesses of His presence in the world.
\end{abstract}

Keywords: Jesus Christ, consecrated life, consecration, witness, holiness, vocation, faith, Church

Fin dall'inizio della vita consacra Gesù Cristo era considerato come il fondatore e il modello delle persone consacrate. Il fondamento di questa considerazione proveniva dalle sorgenti cristologiche della vita consacrata cioè prima di tutto dall'esempio stesso della vita del Signore.

In questo articolo, diviso in tre parti, in primo piano sta la persona di Cristo, che emerge come il vero consacrato a Dio e modello di tutti coloro che vogliono offrirsi a Dio. Il primo paragrafo vuole mostrare Gesù sotto la luce dei consigli evangelici, che lui ha scelto e ha praticato durante la sua vita terrena nella prospettiva della sua missione, cioè compiere il disegno di salvezza. Il paragrafo seguente sviluppa il tema della dimensione pasquale della vita consacrata concentrandosi sul battesimo, come inizio della vita divina dell'uomo, sull'Eucaristia e sui consigli evangelici considerati nell'aspetto pasquale. Da queste due parti nasce il tema della testimonianza della persona di Cristo nella vita quotidiana dei consacrati. La vita stessa delle persone consacrate, costruita sulla castità, povertà ed obbedienza, e la missione di annunciare Cristo vi emergono come punti centrali della testimonianza del Signore. 


\section{I consacrati come Cristo per il Regno di Dio}

La prospettiva cristocentrica è una delle linee dominanti e più evidenti nella storia della vita consacrata. Lo è anche nell'Esortazione Vita Consacrata, la quale mostra Gesù come il Consacrato a Dio per eccellenza ${ }^{1}$. È possibile dunque intendere bene il documento soltanto attraverso la lettura completa e progressiva della sua cristologia ${ }^{2}$.

Il raccordo tra l'aspetto trinitario e quello cristologico è inerente allo stesso mistero cristiano. Ogni forma di vita cristiana trova in Gesù la via di comunione con il Padre nello Spirito. F. Ciardi afferma questo dicendo che „Cristo è infatti la piena rivelazione del Dio trinitario: lo manifesta e lo comunica. Aderire a lui è entrare nel dinamismo della vita della Trinità"3.

La figura di Cristo appare al centro di ogni riflessione ed il volto divino-umano del Signore diventa l'icona fondante di tutto il documento dall'inizio alla fine. Questa concentrazione cristologica - secondo J. Castellano Cervera - vuol dire „speciale rapporto con Cristo e per lui con il Padre e lo Spirito Santo, con colui che è l'inviato del Padre, consacrato nello Spirito, alla cui immagine si configura e alla cui sequela si modella la vita consacrata" ${ }^{4}$. Da questa ottica derivano alcuni temi fondamentali come la sequela totale, connotata dai consigli evangelici, loblazione

1 „Tante persone hanno cercato, con la parola e con l'azione, di incarnare il Vangelo nella propria esistenza, per riproporre nel loro tempo la viva presenza di Gesù, il Consacrato per eccellenza e l'apostolo del Padre". Giovanni PAOLO II, Esortazione apostolica Vita consecrata, Roma 1996 (=VC), 9.

2 Cf, A. Amato, Il volto di Gesù Cristo, il consacrato e il missionario del Padre nella vita consacrata, in: Conferenza Italiana Superiori Maggiori (red.), Consacrati da Dio, dono alla Chiesa e al mondo. Approfondimenti sull'Esortazione "Vita Consecrata», Roma 1997, 147-166; J. Castellano Cervera, Il mistero e la missione di Cristo nella vita consacrata, „Vita Consacrata” 3-4(32) (1996), 342; B. SEcondin, Il profumo di Betania. La vita consacrata come mistica, profezia, terapia, Bologna 1997, 56-65.

${ }^{3}$ F. CIARDI, Il radicamento evangelico della vita consacrata e il suo sviluppo storico «in ascolto dello Spirito", in: Conferenza Italiana Superiori Maggiori (red.), Consacrati da Dio, dono alla Chiesa e al mondo..., op. cit., 97.

4 J. Castellano Cervera, Dimensione teologica e spirituale della vita consacrata: tradizione, novità, profezia, in: Vita Consecrata. Studi e riflessioni, Roma 1996, 44. 
dell'intera vita, lofferta di tutta la persona a sua imitazione, per la missione, lorientamento per il Regno. Queste nozioni corrispondono a quella forma di vita di Gesù vissuta come un esodo verso il Padre. Anche nell'altro articolo, lo stesso autore presenta come tema centrale dell'Esortazione e filo conduttore proprio il suo carattere fortemente cristologico, che nella sua dimensione trinitaria, ecclesiale e sociale, struttura la vita consacrata. Come affermazione di queste parole, si riferisce al primo punto del documento, dove il Papa dice dellorigine evangelica della vita consacrata: ,profondamente radicata negli esempi e negli insegnamenti di Cristo Signore” e la definisce come la manifestazione dei „tratti caratteristici di Gesù"'.

Armando Bandera sviluppa questo pensiero dicendo che si deve dare rilievo non soltanto alle parole di Gesù, ma anche al suo modo d'agire. Gli esempi di vita del Signore equivalgono alle sue opere. Quindi, „per comprendere correttamente le parole di Gesù sui consigli evangelici, è necessario leggere il Nuovo Testamento alla luce del quadro concreto di vita che Gesù scelse per se stesso"'. Gli esempi che Gesù ha lasciato, il suo modo di vita sono dunque il vero fondamento biblico della vita consacrata.

Gesù Cristo per primo, nella sua esistenza terrena, ha perfettamente incarnato l'obbedienza al Padre, la verginità e la povertà. Le persone consacrate sono chiamate all'intimità con lui ed alla imitazione del Figlio di Dio. „La vita consacrata «più fedelmente imita e continuamente rappresenta nella Chiesa», per impulso dello Spirito Santo, la forma di vita che Gesù, supremo consacrato e missionario del Padre per il suo Regno, ha abbracciato ed ha proposto ai discepoli che lo seguivano (cf. Mt 4,18-22; Mc 1,16-20; Lc 5,10-11; Gv 15,16)"7.

Nel Vangelo vediamo Gesù come colui che è stato consacrato dal Padre per compiere la sua missione. L'Esortazione pone l'accento sul fatto

${ }^{5}$ VC 1. Cf. J. CAstellano Cervera, Il mistero e la missione di Cristo nella vita consacrata, op. cit., 341-343.

6 Bandera A., Vita Consecrata: Il numero iniziale, „Vita Consacrata” 3(33) (1997), 255.

7 VC 22. Cf. Concilio Vaticano II, Costituzione dogmatica sulla Chiesa Lumen gentium, Roma 1964 (=LG) 44. 
che con la consacrazione a Dio è legata sempre una missione da compiere, un disegno divino. Il testo continua: „Gesù stesso, infatti, è colui che «Dio ha consacrato in Spirito Santo e potenza» (At 10,38), «colui che il Padre ha consacrato e mandato nel mondo» (Gv 10,36). Accogliendo la consacrazione del Padre, il Figlio a sua volta si consacra a Lui per l'umanità (cr. Gv 17,19): la sua vita di verginità, di obbedienza e di povertà esprime la sua filiale e totale adesione al disegno del Padre (cf. Gv 10,30; 14,11)"8.

In questo testo vediamo Cristo nella sua relazione, tanto con il Padre e lo Spirito Santo, quanto con l'umanità. Fare la volontà di Dio e missione di salvezza agli uomini è la cosa primaria e più importante in tutta la sua missione. „Egli è l'obbediente per eccellenza, disceso dal cielo non per fare la sua volontà, ma la volontà di Colui che lo ha mandato (cf. Gv 6,38; Eb 10,5.7)"'. È evidente, perciò, che la vita consacrata trova il suo contenuto centrale e il modello perfetto nel Cristo. È lui che chiama alla sua particolare intimità e rende capaci di vivere in questo suo modo. Per questo il documento dice che „la vita consacrata costituisce memoria vivente del modo di esistere e di agire di Gesù come Verbo incarnato di fronte al Padre e di fronte ai fratelli" ${ }^{10}$. Questa memoria diventa la tradizione vivente della sua persona, della sua vita e del suo messaggio. Ogni religioso deve cercare il modo più esplicito di vivere in Cristo e sperimentare Cristo in se stesso. Il frutto della formazione dei consacrati è perciò un vitale rapporto con il Signore. Questo porta anche a comunicare i sentimenti di Gesù verso il prossimo e verso il mondo ${ }^{11}$.

Quando l'Esortazione Vita Consecrata comincia a parlare sulle sorgenti cristologiche della vita consacrata si serve della icona della Trasfigurazione. Già nel primo capitolo afferma che il fondamento evangelico di questa forma di vita si deve cercare nel rapporto speciale che Gesù stabilì con alcuni dei suoi discepoli, invitandoli a porre la propria vita a servizio di Regno di Dio, lasciando tutto e imitando da vicino la sua

8 VC 22.

9 VC 22. Cf. Bandera A., Un silenzio e un clamore, „Vita Consacrata” 3-4(32) (1996), 328-329.

$10 \mathrm{VC} 22$.

11 Cf. Concilio Vaticano II, Decreto sul rinnovamento della vita religiosa Perfectae caritatis, Roma 1965 (=PC), 1; A. PIGNA, I contenuti, „Vita Consacrata” 3-4(32) (1996), 297. 
forma di vita ${ }^{12}$. Alcuni autori, come per esempio Jean Galot, accentuano che l'icona del Cristo trasfigurato usata nel documento aiuta a comprendere meglio la natura stessa della vita consacrata. „Laccento posto sulla Trasfigurazione risponde a un desiderio profondo dei chiamati alla vita consacrata: che la loro possa essere una vita trasfigurata!"'3. Questo non significa però che soltanto $i$ consacrati sono chiamati a entrare insieme con Gesù nel mistero della Trasfigurazione. Ogni vita cristiana è destinata a essere trasfigurata. La speciale chiamata di Gesù a seguire la sua forma di vita significa una partecipazione più intensa nel mistero di Trasfigurazione. Questa chiamata è nello stesso tempo la introduzione nella sua intimità e l'invito a vivere più radicalmente l'ideale evangelico. Quando la persona chiamata da Dio decide di entrare in questo patto damore, fa presente nella sua vita il mistero di Cristo trasfigurato, lo partecipa con tutto il cuore, con tutta l'anima e con tutte le forze. Così, come vediamo anche nel Vangelo nell'esempio dei tre apostoli prescelti: Pietro, Giacomo e Giovanni, la dedizione completa alla persona di Gesù, in vista della dedizione totale alla sua opera di salvezza, diventa la prima forma di vita consacrata, istituita e promossa da Cristo stesso. Galot afferma che „da questa forma iniziale di vita consacrata, scaturiranno da una parte le forme di vita dei sacerdoti, legate al ministero sacerdotale, e, dall'altra, le molteplici forme di vita consacrata non legate al ministero ordinato: vita religiosa o vie dei consigli evangelici secondo diverse modalità" ${ }^{\prime 4}$.

Gesù vuole mostrare la forza di questa sua chiamata creando un popolo nuovo, il gruppo dei dodici. Tutto questo ci mostra la forza della sua chiamata che crea una nuova personalità. Vediamo, dunque, che la chiamata del Signore non cambia il proprio temperamento ed i valori della vita dei apostoli, ma cambia il loro destino, trasforma la loro vita. Tutto questo è stato espresso nel fatto di cambiare di nome nel caso di Pietro, Giacomo e Giovanni (cf. Gv 1,42; Mc 3,17) ${ }^{15}$.

12 Cf. VC 14.

13 J. GAlot, La Trasfigurazione, „Vita Consacrata” 3-4(32) (1996), 351. Cf. P. G. CABRA, Un itinerario spirituale a partire dalle icone, in: Conferenza Italiana Superiori Maggiori (red.), Consacrati da Dio, dono alla Chiesa e al mondo..., op. cit., 115-118.

14 J. Galot, La Trasfigurazione, op. cit., 352.

15 Cf. J. Galot, La Trasfigurazione, op. cit., 352-353. 
La vita e gli esempi di Gesù Cristo sono, quindi, la fonte da cui scaturisce la vita dei consacrati. Lo scopo di questa vita è la configurazione a Cristo. Questo è il nucleo centrale da cui sgorga, fiorisce e si sviluppa la vita consacrata: „Per me vivere è Cristo” (Fil 1,21; Gal 2,20). Gesù stesso ha inaugurato, iniziato e fondato questa nuova forma di vita, l'ha scelto per sé e per sua Madre e l'ha proposta ad alcuni che ha scelto e ha invitato a lasciare tutti i propri beni e la famiglia per seguirlo. Senza dubbio, questa scelta che ha fatto Gesù non era casuale. Lui si è fatto un esempio per quelli che hanno ricevuto questa speciale vocazione ${ }^{16}$.

Dicendo speciale vocazione non si nega che Cristo è modello di santità per tutti gli uomini e non soltanto i cristiani. È chiaro pure che non tutti gli aspetti della vita terrena di Gesù siano applicabili ad ogni cristiano. Jean Galot così chiarisce la nozione di speciale sequela: „Ognuno è invitato a imitare Gesù secondo la propria vocazione particolare. Così, non tutti sono chiamati a «prendere Cristo come sola ricchezza», nel senso di una rinuncia totale ai beni. Tutti i cristiani hanno il dovere di evitare l'attaccamento alle ricchezze, di rinunciare a ogni bramosia del denaro; ma essi non sono invitati tutti da Cristo a disfarsi dei propri beni” ${ }^{17}$.

Quindi possiamo dire che la speciale sequela di Cristo, che è nucleo della vita consacrata, significa seguire Gesù, stare con lui, dedicarsi a lui e, come lui, al servizio del Padre e dei fratelli. J. Castellano pone l'accento sulladesione totale a Gesù: „Anche se tutti nella Chiesa sono chiamati a vivere il mistero di Cristo, l'impegno totale della vita mediante i consigli evangelici contrassegna lesistenza di tutti i chiamati a unade-

${ }^{16}$ Nella teologia precedente si discuteva sulla fondazione della vita consacrata da parte di Gesù. Questa discussione ha trovato il suo fine nella seguente dichiarazione del Papa: "Gesù stesso, chiamando alcune persone ad abbandonare tutto per seguirlo, ha inaugurato questo genere di vita che, sotto l'azione dello Spirito Santo, si svilupperà gradualmente lungo i secoli nelle varie forme della vita consacrata". VC 29c. Per approfondire questo tema vedi: F. CIARDI, Il radicamento evangelico della vita consacrata e il suo sviluppo storico «in ascolto dello Spirito", op. cit., 102-105; J. GALOT, Cristo fondatore della vita consacrata, „Vita Consacrata” 4(27) (1991), 306-315; A. PIGNA, Risposta della esortazione alle questioni poste dal sinodo, in: Conferenza Italiana Superiori Maggiori (red.), Consacrati da Dio, dono alla Chiesa e al mondo..., op. cit., 187-201.

${ }^{17} \mathrm{~J}$. GALOT, Cristo fondatore della vita consacrata, op. cit., 312. 
sione totale alla persona di Gesù e a conformare la propria esistenza alla sua"18.

La natura della formazione nella vita consacrata è pure cristocentrica. I consacrati hanno lasciato ogni cosa per amore di Cristo e lo vogliono seguire come l'unica cosa necessaria ${ }^{19}$. L'Esortazione dedica al tema della formazione i numeri 65-71 mostrando le sue diverse tappe. Per la formazione il documento intende ,un processo vitale, attraverso il quale la persona si converte al Verbo di Dio fin nelle profondità del suo essere e, nello stesso tempo, impara l'arte di cercare i segni di Dio nelle realtà del mondo" 20 . I religiosi vogliono prendere e imitare la forma di vita del Signore per trasformarsi in persone cristoformi attraverso l'immedesimazione dei suoi atteggiamenti e il coinvolgimento pieno nella sua missione. Attraverso lo sforzo di rivestirsi „del Signore Gesù Cristo” $(\mathrm{Rm} 13,14)$ vogliono imitare e vivere in questa forma di vita, che egli abbracciò. Il cercare Gesù e l'aderire a lui con tutta la mente e con tutto il cuore è lo scopo ultimo e l'impegno di tutta la loro vita ${ }^{21}$.

Possiamo a questo punto domandare cosa significa l'espressione „esistenza cristoforme" usata nel punto 14 dell'Esortazione? Il Papa scrive: „Il fondamento evangelico della vita consacrata va cercato nel rapporto speciale che Gesù, nella sua esistenza terrena, stabilì con alcuni dei suoi discepoli, invitandoli non solo ad accogliere il Regno di Dio nella propria esistenza a servizio di questa causa, lasciando tutto e imitando da vicino la sua forma di vita. Una tale esistenza "cristoforme», proposta a tanti battezzati lungo la storia, è possibile solo sulla base di una speciale vocazione e in forza di un peculiare dono dello Spirito"22_

Nella luce della Trasfigurazione vediamo che questa formulazione esprime unesistenza che assume la forma propria dell'esistenza di Cristo. Durante il battesimo viene impressa nellanima di ogni cristiano una

18 J. Castellano Cervera, Il mistero e la missione di Cristo nella vita consacrata, op. cit., 344. Cf. VC 1.

19 Cf. PC 5.

$20 \mathrm{VC} 68$.

${ }^{21}$ Cf. VC 14; LG 46; B. GoyA, Formazione integrale alla vita consacrata alla luce della esortazione post-sinodale, Bologna 1997, 20-22.

$22 \mathrm{VC} 14$. 
somiglianza o „configurazione” a Cristo. Tutti sono chiamati a imitare Gesù, a conformare la propria vita a lui, alla santità che implica consacrazione e configurazione. Alcuni però sono chiamati a rispondere all'invito speciale di Cristo: „seguimi” e vogliono scegliere una via peculiare del celibato o della verginità, diventando così più simili al Signore, che ha rinunciato al matrimonio e ha vissuto la sua vita nella totale oblazione al Padre e nel servizio al Regno di Dio. Nella vita di queste persone „la consacrazione battesimale è portata ad una risposta radicale nella sequela di Cristo mediante l'assunzione dei consigli evangelici, primo ed essenziale tra esse il vincolo sacro della castità per il Regno dei Cieli”23.

Gesù dona se stesso al Padre: la sua persona, la sua intera esistenza, il suo servizio. Lo specchio più chiaro di questa totalità del suo dono è la Croce. La misura della dedizione del Signore è la ricchezza dell'amore di $\mathrm{Dio}^{24}$. La vita consacrata è compresa come un dono di conformazione a Cristo fatto dalla Trinità alla Chiesa e ricevuto gratuitamente nella speciale chiamata di Dio. Il dono divino offerto alle persone consacrate esige la risposta la quale è un totalizzante e deciso si iniziale alla sua proposta e al suo invito. La scelta di Gesù è assimilata e accolta in modo più pieno quando la persona lo confessa per la sua vita e quando ogni altra realtà va commisurata su di Gesù. La parola „totalizzante” che frequentemente viene usata nel documento, significa - secondo P. G. Cabra - uno sforzo, „una forte tensione spirituale nell'intento di conformarsi a Cristo vergine, povero e obbediente, non solo esternamente, ma anche in un processo di interiorizzazione" 25 .

Il Signore ha scelto il modo di vita nella castità, povertà ed obbedienza per realizzare l'opera della redenzione, del cui contenuto essi

23 VC 14. Cf. IX Sinodo dei Vescovi, La vita consacrata. Proposte, Roma 1994, in: Enchiridion Vaticanum T. 14, Bologna 1997, 3; A. Amato, Spunti di lettura cristologico-trinitaria della Vita Consecrata, in: Vita Consecrata. Una prima lettura teologica, Milano 1996, 37-38.

${ }^{24}$ Cf. B. Maggioni, Il fondamento evangelico della vita consacrata, in: Vita consacrata un dono del Signore alla sua Chiesa, Leumann (Torino) 1994, 95-98.

25 P. G. CABRA, I consigli evangelici un dono per la missione, „Vita Consacrata” 6(32) (1996), 594. Non si la intende quindi come una sorta di totalitarismo, il quale esclude la libera scelta dell'uomo. Cf. F. CIARDI, Il radicamento evangelico della vita consacrata e il suo sviluppo storico «in ascolto dello Spirito», op. cit., 97-101. 
fanno parte. La specifica vocazione dei consacrati come pure l'insieme della loro vita nella Chiesa e nel mondo "attingono il loro carattere e la forza spirituale dalla profondità stessa del mistero della redenzione" ${ }^{26}$. I consigli evangelici appaiono qui come elementi chiave nel programma di quella vita alla quale Cristo chiama le persone consacrate dicendo: seguimi. Da esse viene una particolare caratteristica e specifico segno dell'economia della redenzione ${ }^{27}$.

Il significato più profondo di imitare e seguire Gesù è fare di lui il centro della propria esistenza, vivere per lui e lasciarsi guidare da lui. Vediamo questi elementi caratteristici nella vita dei apostoli per i quali il Cristo è stato il centro della loro esistenza. San Paolo dice ai Filippesi: „Per me infatti il vivere è Cristo e il morire un guadagno” (Fil 1,21) ${ }^{28}$. Nell'Esortazione prevale il concetto paolino dell'imitazione di Cristo, quindi quello della sequela. Non si tratta qui di una semplice imitazione solo esterna o di avere i comportamenti di Cristo. La vera imitazione del Signore, la sequela, consiste nell'identificazione personale ed esistenziale, per la quale Cristo viene spiritualmente presente nella persona del consacrato ${ }^{29}$.

La sequela e l'imitazione sono due concetti, contenuti e realtà diversi. B. Proietti spiega questo in modo seguente: „Sequela implica un cammino esteriore, fatto di gesti e di decisioni, che esprimono chiaramente un camminare dietro a Gesù. Imitazione, invece, richiama lo sforzo morale (e mistico) per riprodurre i tratti di Gesù visto come modello di tutta la vita cristiana (nella povertà, obbedienza, umiltà ecc.). La sequela tro-

${ }^{26}$ Giovanni Paolo II, Esortazione apostolica Redemptionis donum, Roma 1984 (=RD), 1 .

${ }_{27}$ Cf. VC 90c; RD 9; A. BAndera, Magistero postconciliare, in: Dizionario Teologico della Vita Consacrata, Milano 1994, 918-919.

${ }^{28}$ Cf. VC 15; J. GAlot, La Trasfigurazione, op. cit., 353-354; P. G. CABRA, I consigli evangelici un dono per la missione, op. cit., 594.

${ }^{29}$ Cf. J. A. Estrada Díaz, Imitazione di Gesù Cristo, in: Dizionario Teologico della Vita Consacrata, op. cit., 839. Secondo l'autore: „in realtà, il concetto paolino di imitazione stabilisce una connessione fra l'incarnazione del Verbo e l'innabitazione dello Spirito, poiché il Pneuma ci unge, ci rende simili a Cristo cristianizzandoci e ci porta ad assumerlo come modello". 
va il suo vertice nel gesto di abbandonare tutto per rispondere alla chiamata di Gesù, l'imitazione culmina nel fatto di intrattenere con il Padre una relazione analoga a quella di Gesù"30.

La chiamata universale a seguire Gesù vedendo in lui il senso ultimo della propria vita è, quindi, evidente in modo particolare nei consacrati. Nella loro esperienza del Signore è molto forte la risonanza delle parole di Pietro: „Signore, è bello stare qui!” (Mt 17,4). Giovanni Paolo II afferma che "queste parole dicono la tensione cristocentrica di tutta la vita cristiana. Esse, tuttavia, esprimono con particolare eloquenza il carattere totalizzante che costituisce il dinamismo profondo della vocazione alla vita consacrata: «Come è bello restare con Te, dedicarci a Te, concentrare in modo esclusivo la nostra esistenza su di Te!» In effetti, chi ha ricevuto la grazia di questa speciale comunione di amore con Cristo, si sente come rapito dal suo fulgore: Egli è il «più bello tra i figli dell'uomo» (Sal 44(45),3), l'Incomparabile"31.

Il rapimento di Pietro manifesta questo peculiare attaccamento alla persona di Gesù Cristo nella vita consacrata. Nella loro vita, le persone consacrate vogliono conservare la presenza del Signore, non per chiedere alcun favore particolare come fanno altri, ma semplicemente perché questa presenza colma i loro desideri e li riempie di una gioia profonda. I consacrati, come Pietro, sperimentano che Cristo è il bene supremo per la loro vita e per la vita di ogni uomo. Da questo nasce anche il desiderio di poter godere questa presenza del Maestro che li ha chiamati a vivere nella sua intimità. L'esperienza della gioia profonda, che scaturisce dalla presenza costante del Cristo, esprime in modo più pieno il carattere totalizzante della vita consacrata di cui parla l'Esortazione ${ }^{32}$.

Il cristocentrismo della vita consacrata diventa anche una vera e propria assolutizzazione di Cristo nella vita dei consacrati. Egli è il centro, il tutto della vita, senza nessuna mediazione umana. L'essere consacra-

${ }^{30}$ B. Proietti, Sequela Christi e imitazione, in: Dizionario degli istituti di perfezione, VIII, Roma 1988, 1287.

31 VC 15. Cf. A. Амato, Il volto di Gesù Cristo..., op. cit., 152-154.

32 Cf. A. Amato, Spunti di lettura cristologico-trinitaria della Vita Consecrata, op. cit., 42-45; J. GALOT, La Trasfigurazione, op. cit., 357. 
to significa dunque confessare e riconoscere Gesù come Figlio di Dio creatore, redentore, santificatore e personale datore di senso e di valore alla propria persona. Le persone consacrate adorano Gesù nell'Eucaristia, nella Parola, nella Chiesa, nei sacramenti e nella comunità. Si crea così una vera comunione con lui, un novo legame che nasce dalla ricerca della volontà di Dio. Questa unità con Cristo e così forte che il Papa alla fine dell'Esortazione conclude usando parole molto significative: „Non dimenticate che voi, in modo particolarissimo, potete e dovete dire non solo che siete di Cristo, ma che "siete divenuti Cristo»!"33.

Il Papa chiude il documento con una forte affermazione sulla gratuità della vita consacrata, come una restituzione della vita a colui che l'ha donata per noi. Servendosi dell'eloquente icona dell'unzione a Betania, ancora una volta pone l'accento sulla priorità della dedicazione alla persona di Gesù e sottolinea il valore supremo di questa gratuità amorosa che possiamo ammirare nel gesto di Maria di Betania. Anche qui, al centro è la persona di Cristo, sommamente amata, al di là di qualsiasi interesse o funzionalità. „A chi è concesso il dono inestimabile di seguire più da vicino il Signore Gesù appare ovvio che Egli possa e debba essere amato con cuore indiviso, che a Lui si possa dedicare tutta la vita e non solo alcuni gesti o alcuni momenti o alcune attivita"' ${ }^{34}$. Nel gesto irrazionale di Maria i consacrati vedono il nucleo della propria donazione al Signore. La risposta di Gesù che apprezza quel gesto è spiegabile solo con la logica dell'amore, anche se non tutti la comprendono e lapprezzano ${ }^{35}$.

La vita consacrata definita come una speciale sequela di Cristo è una particolare comunione d'amore con il Signore. Lui diventa il centro della vita. Questo modo di vivere accoglie in maniera peculiare il mistero del Figlio di Dio e rispecchia il suo stesso vivere. In conseguenza, la vita consacrata esprime ciò a cui ogni forma di vita cristiana è chiamata: rivivere il mistero di Cristo.

${ }^{33}$ VC 109. Cf. A. Amato, Spunti di lettura cristologico-trinitaria della Vita Consecrata, op. cit., 38-41.

34 VC 104. Cf. A. Амato, Il volto di Gesù Cristo..., op. cit., 165-166; J. Castellano Cervera, Il mistero e la missione di Cristo nella vita consacrata, op. cit., 349.

35 Cf. P. G. CABRA, Un itinerario spirituale a partire dalle icone, op. cit., 141-144. 


\section{Dimensione pasquale della vita consacrata}

La dimensione pasquale della vita consacrata è stata sottolineata nella Esortazione soprattutto nei punti 23 e 24. Il Papa mostra Gesù Crocifisso, dal quale emergono tutte le vocazioni insieme con il dono fondamentale dello Spirito Santo: „I discepoli e le discepole sono invitati a contemplare Gesù esaltato sulla Croce, dalla quale «il Verbo uscito dal silenzio», nel suo silenzio e nella sua solitudine, afferma profeticamente l'assoluta trascendenza di Dio su tutti i beni creati, vince nella sua carne il nostro peccato e attira a sé ogni uomo e ogni donna, donando a ciascuno la nuova vita della risurrezione (cf. Gv 12,32; 19,34.37). Nella contemplazione di Cristo crocifisso trovano ispirazione tutte le vocazioni; da essa traggono origine, con il dono fondamentale dello Spirito, tutti i doni e in particolare il dono della vita consacrata" ${ }^{36}$.

Fabio Ciardi osserva che nel mistero della Croce di Gesù viene rivelata la pienezza dell'amore. Cristo mostra il suo amore tutto aperto e tutto donato. Sulla Croce, nella sua estrema inattività, Gesù compie la sua missione di redenzione. „Lì, privato della comunione con il $\mathrm{Pa}$ dre e nella solitudine più profonda, esprime la più alta contemplazione. Lì compie la più grande opera di misericordia aprendo il paradiso al ladrone pentito" 37 .

Il Papa menziona la persona dell'Apostolo Giovanni che, accanto a Maria, essendo toccato dall'amore straordinario di Dio, diventa uno tra tanti altri che „si sentono chiamati a seguire l'Agnello immolato e vivente, dovunque Egli vada (cf. Ap 14,1-5)"38. Come discepolo amato, le persone consacrate, unendosi con Gesù Crocifisso, fanno l'esperienza di Dio-Amore in modo pieno ed immediato. La bellezza del Cristo Trasfigurato raggiunge la sua dimensione più profonda. Il Volto del Signore Crocifisso risplende qui una nuova bellezza: quella dell'Amore. „Colui che nella sua morte appare agli occhi umani sfigurato e sen-

36 VC 23b.

37 F. CIARDI, Il radicamento evangelico della vita consacrata e il suo sviluppo storico «in ascolto dello Spirito», op. cit., 110.

38 VC23. Cf. P. G. CABRA, Un itinerario spirituale a partire dalle icone, op. cit., 118-121. 
za bellezza tanto da indurre gli astanti a coprirsi il volto (cf. Is 53,2-3), proprio sulla Croce manifesta pienamente la bellezza e la potenza dell'amore di Dio"39.

Lespressione sequela Christi, che si usa spesso a riguardo della vita consacrata, non significa semplicemente camminare sui passi di Gesù o imitarlo. Essa significa entrare nel suo mistero di morte e di vita ed esprimerlo nel proprio intimo e nella propria condotta. Ponendo al centro il mistero del Cristo Crocifisso, la vita consacrata in modo peculiare contribuisce a tener viva nella Chiesa la profonda coscienza che la Croce di Gesù è la sovrabbondanza dellamore di Dio che trabocca su questo mondo. L'Esortazione sottolinea anche le difficoltà e le prove nella vita dei consacrati nelle quali si testimonia la loro unità con il Maestro ${ }^{40}$.

Nel cammino dell'imitazione di Cristo il battesimo è un primo segno della dimensione pasquale del cristianesimo e della vita consacrata. I religiosi, nella linea del battesimo, rafforzano il titolo per cui ogni cristiano è alter Christus, riproduzione viva di Cristo ${ }^{41}$. La vita del cristiano è legata al mistero pasquale di Cristo. Vivere nel Cristo significa condurre una esistenza pasquale, percorrere la via al Padre che Gesù ha aperto sulla Croce. Il battesimo sta all'inizio di questa via di salvezza e introduce il fedele nel mistero della redenzione. Egli, come scrive Ignazio Sanna, „vi resta in modo stabile e non cessa di celebrare la sua unione a Cristo

${ }^{39} \mathrm{VC} 24$.

40 Cf. VC 24. Il Papa scrive che: „La loro fedeltà all'unico Amore si mostra e si tempra nell'umiltà di una vita nascosta, nell'accettazione delle sofferenze per completare ciò che nella propria carne «manca ai patimenti di Cristo» (Col 1,24), nel sacrificio silenzioso, nell'abbandono alla santa volontà di Dio, nella serena fedeltà anche di fronte al declino delle forze e della propria autorevolezza. Dalla fedeltà a Dio scaturisce pure la dedizione al prossimo, che le persone consacrate vivono non senza sacrificio nella costante intercessione per le necessità dei fratelli, nel generoso servizio ai poveri e agli ammalati, nella condivisione delle difficoltà altrui, nella sollecita partecipazione alle preoccupazioni e alle prove della Chiesa”. VC 24.

${ }^{41}$ Cf. E. Gambari, Manuale della vita religiosa, Roma 1970, 143. Sulla relazione fra battesimo e la vita consacrata: J. Gribomont, Professione e battesimo, in: Dizionario degli istituti di perfezione, VII, Roma 1983, 890-892; G. LECLERCQ, Professione religiosa secondo battesimo, in: „Vita Religiosa” 1 (1967), 3-8; C. PAlmés DE Genover, Battesimo, in: Dizionario Teologico della Vita Consacrata, op. cit., 120-133. 
nella morte e nella glorificazione fino al giorno in cui essa sarà completa, quando egli si addormenterà con Cristo nella morte $(2 \mathrm{Tm} 2,11)$, e con lui risusciterà nel giorno finale $(\mathrm{Rm} 6,8)^{\prime \prime 42}$. L'immergersi nell'acqua esprime l'atto di morire ed essere sepolto con Cristo. Il riemergere dall'acqua e considerato come risuscitare con Cristo alla vita nuova. Da questa configurazione e comunione a Cristo fluisce la figliolanza divina, dono dello Spirito, la vita in Cristo ormai liberata dal peso della legge e dalla minaccia del peccato. L'Apostolo Paolo esprime tutto questo nella Lettera ai Romani: „O non sapete che quanti siamo stati battezzati in Cristo Gesù, siamo stati battezzati nella sua morte? Per mezzo del battesimo siamo stati dunque sepolti insieme a lui nella morte, perché come Cristo fu risuscitato dai morti per mezzo della gloria del Padre, così anche noi possiamo camminare in una vita nuova" $(\mathrm{Rm} 6,3-4)^{43}$.

La vocazione alla vita consacrata è una confermazione della consacrazione battesimale, la quale è portata ad una risposta più radicale nella sequela di Cristo mediante dei consigli evangelici ${ }^{44}$. Quindi, tra il battesimo e la professione religiosa non cè nessuna tensione. La professione suppone sempre il battesimo e non lo sostituisce, è semplicemente una rinnovazione del battesimo. Il consacrato che vive secondo i consigli evangelici adempie in modo più stretto le promesse del suo battesimo. L'Esortazione esprime questo in modo molto chiaro: „Nella tradizione della Chiesa la professione religiosa viene considerata come un singolare e fecondo approfondimento della consacrazione battesimale in quanto, per suo mezzo, l'intima unione con Cristo, già inaugurata col battesimo, si sviluppa nel dono di una conformazione più compiutamente espressa e realizzata, attraverso la professione dei consigli evangelici" ${ }^{35}$. La vita religiosa implica per questo una vera vita cristiana. D’altra parte, benché la professione è unespressione più perfetta del battesimo, tuttavia sem-

${ }^{42}$ I. Sanna, Mistero pasquale, in: S. De Fiores, T. Goffi (red.), Nuovo dizionario di spiritualità, Cinisello Balsamo (MI) 1999, 979.

${ }^{43}$ Cf. J. Castellano Cervera, Battesimo, in: Dizionario enciclopedico di spiritualità, Roma 1995, 280-281.

${ }^{44}$ Cf. VC 14.

${ }^{45}$ VC 30. Cf. PC 5; RD 7; G. LeClencQ, Professione religiosa secondo battesimo, op. cit., 3; C. PALmÉs de Genover, Battesimo, op. cit., 120-125. 
pre costituisce una nuova consacrazione a Dio con un nuovo e speciale titolo $^{46}$. Il Papa dice: „Il battesimo non comporta per se stesso la chiamata al celibato o alla verginità, la rinuncia al possesso dei beni, l'obbedienza ad un superiore, nella forma propria dei consigli evangelici. Pertanto la professione di ultimi suppone un particolare dono di Dio non concesso a tutti, come Gesù stesso sottolinea per il caso del celibato volontario (cf. Mt 19,10-12)" ${ }^{47}$. È necessario dunque conservare la distinzione fra l'una ed l'altra consacrazione ${ }^{48}$. Carlos Palmés de Genover racchiude questa distinzione nelle tre affermazioni: „La consacrazione propria della vita religiosa non è essenzialmente distinta dalla consacrazione battesimale. È una ratificazione della medesima. (...) La consacrazione religiosa è peculiare, con una peculiarità che non si riferisce al contenuto, bensì ai mezzi per vivere il battesimo. (...) Questi mezzi aiutano il cristiano che opta per la vita religiosa a esprimere con maggior pienez$z a$ la propria consacrazione battesimale, a viverla con maggior coerenza e profondità" 49 .

Come il battesimo sommerge i cristiani nella morte di Cristo così la consacrazione religiosa sommerge la persona consacrata nel mistero pasquale di Gesù. L'unione con il Signore nella morte si esprime nel vivere l'abnegazione nella peculiare sequela di Cristo, la quale si realizza nel celibato, nella vita comunitaria, nella povertà, obbedienza e missione. L'abnegazione è quindi una disposizione generale dell'uomo che facilità la pratica di tutte le virtù, in tutto ciò che hanno di contrario all'egoismo $\mathrm{o}$ all'amor proprio disordinato. Vediamo perciò che l'essenziale è sostituire gli interessi personali con quelli del Regno.

L'identificazione con Gesù risorto pone l'accento sul senso escatologico della vita consacrata. Il religioso come l'uomo nuovo, sentendosi figlio di Dio, diventa il testimone della città di Dio. Continuando il suo

\footnotetext{
${ }^{46}$ Cf. LG 44.

47 VC 30.

48 Cf. J. Galot, Consacrazione battesimale e consacrazione religiosa, „Vita Consacrata” 12(14) (1978), 592-598.

49 Cf. P. G. CABra, La missione nella Esortazione apostolica, in: Vita Consecrata. Studi e riflessioni, op. cit., 189-193; J. GALOT, Cristo fondatore della vita consacrata, op. cit., 309-311; C. Palmés de Genover, Battesimo, op. cit., 120-121.
} 
pensiero, Carlos Palmés de Genover afferma che il segno caratteristico dell'esperienza del Risorto nella vita dei consacrati è la semplicità e l'allegria. La persona consacrata aiuta agli altri a vedere e a sperimentare la continua azione santificatrice di Dio nella vita e nella storia di ogni cristiano diventando così il testimone della risurrezione $e^{50}$.

La dimensione pasquale della vita consacrata appare anche nell' $\mathrm{Eu}$ caristia. Essa „è il sacramento della Pasqua e il mistero pasquale per eccellenza ed implica una spiritualità del cammino" ${ }^{\prime}$. L'Eucaristia unisce il credente alla Croce ed alla morte di Gesù associandolo alla risurrezione. Questo mistero si esprime prima di tutto nelle parole dell'istituzione e nel rito della frazione del pane ${ }^{52}$.

Come centro della vita cristiana e prima di tutto della vita dei consacrati, l'Eucaristia è il modo più profondo dell'unione con Gesù. Nella celebrazione eucaristica le persone consacrate „compiono un atto della Chiesa e per la Chiesa, in comunione con Cristo che si offre al Padre per la salvezza del mondo intero" ${ }^{33}$. La partecipazione quotidiana dei consacrati nel mistero emerge dai cuori delle persone consacrate che vogliono instaurare una comunione sempre più profonda con Cristo, che si fa presente nel mistero della morte e della risurrezione. Il documento pone il forte accento sul fatto che „l'Eucaristia sta per sua natura al centro della vita consacrata, personale e comunitaria. Essa è viatico quotidiano e fonte della spiritualità del singolo e dell'istituto. In essa ogni consacra-

${ }^{50}$ Cf. C. Palmés de Genover, Battesimo, op. cit., 127. Vedi anche: Mantovani M., Un «tempo di gioia»: la formazione iniziale alla vita consacrata, in: P. Vanzan, F. Volpi (red.), Il giubileo e la vita consacrata. Provocazioni e risposte, Roma 2001, 149-161; S. PALUMBIERI, «Gioia» e vita consacrata, in: P. Vanzan, F. Volpi (red.), Il giubileo e la vita consacrata..., op. cit., 111-124.

${ }^{51}$ M. Gesteira Garza, Eucaristia, in: Dizionario Teologico della Vita Consacrata, op. cit., 714 .

${ }^{52}$ Il significato di questo in modo più ampio spiega: I. SANna, Mistero pasquale, op. cit., 980-981. Sull'Eucaristia come sacrificio vedi: J. Castellano Cervera, Eucaristia, in: Dizionario enciclopedico di spiritualità, op. cit., 968-969; M. GesteIra Garza, Eucaristia, op. cit., 705-713; E. Ruffini, Eucaristia, in: S. De Fiores, T. Goffi (red.), Nuovo dizionario di spiritualità, op. cit., 607-610.

${ }^{53} \mathrm{VC} 30$. 
to è chiamato a vivere il mistero pasquale di Cristo, unendosi con Lui nell'offerta della propria vita al Padre mediante lo Spirito" ${ }^{24}$.

Le persone consacrate, come tutti i cristiani, per mezzo dell'Eucaristia partecipano al sacrificio di Gesù, ircorporandovi il sacrificio della propria vita. Questa offerta personale non si riduce a un puro rito esteriore, ma è caratterizzata per l'offerta interiorizzata, sebbene non esclusivamente interiore. Manuel Gesteira Garza scrive: „Così, offrendo la nostra vita intera, tutta l'attività umana, lavoro e gioie, sforzi e sofferenze, diventano spirituali sacrifici graditi a Dio per Gesù Cristo (1 Pt 2,5)"55. La partecipazione al sacrificio del Signore avviene anche per mezzo della comunione dove tutti i cristiani sono invitati a partecipare del suo calice (cf. Mc 10,38). Questa è partecipazione non solo al sangue di Cristo, ma alla sua sorte e al suo destino, quindi nella donazione totale di se stesso. L'offerta della propria vita insieme con Cristo al Padre è atto fondamentale della vita religiosa ${ }^{56}$.

La dimensione pasquale della vita consacrata si esprime in modo speciale nel caso degli consigli evangelici che i consacrati scelgono come via più stretta dell'imitazione di Cristo. Proprio sotto la croce di Gesù vediamo il valore ultimo e le conseguenze estreme dei consigli evangelici nella sua vita: „Lì il suo amore verginale per il Padre e per tutti gli uomini raggiungerà la sua massima espressione; la sua povertà arriverà allo spogliamento di tutto; la sua obbedienza fino al dono della vita" ${ }^{57}$. La vita consacrata viene, quindi, verificata in modo più autentico nella prospettiva della croce.

La consacrazione mediante i consigli evangelici è un inserimento nella filiazione divina di Gesù. Imitando i suoi atteggiamenti filiali per rispondere al dono del Padre, i consacrati si uniscono, per amore a lui, al suo sacrificio. Come nella sua morte Gesù è donato, santificato e consacrato, anche i suoi discepoli che vogliono imitarlo in modo più stretto sono consacrati, santificati nel donarsi al Padre. Jean Beyer così descri-

\footnotetext{
54 VC 95.

55 M. Gesteira Garza, Eucaristia, op. cit., 715.

56 Cf. ibid., 715.

57 VC 23.
} 
ve questa relazione: „Per la sua morte volontaria Gesù è donato, santificato e consacrato affinché coloro che non sono che una cosa sola con lui siano anchessi santificati, consacrati nel donarsi al Padre (Gv 17,19)"58.

Per gli consigli evangelici la persona si consacra a Dio in Cristo come proprietà esclusiva. Questa consacrazione viene considerata come una nuova sepoltura nella morte di Cristo diversa da quella battesimale. La novità consiste in questo che essa si compie mediante l'amore e la speciale vocazione e, in particolare, perché essa effettua un'incessante conversione che porta l'uomo a camminare come Cristo in una vita nuova. Nella consacrazione per i consigli evangelici viene deposto l'uomo vecchio e rivestito l'uomo nuovo, creato secondo Dio nella giustizia e nella santità vera (cf. Ef 4,22.24) $)^{59}$.

L'evento della Trasfigurazione sul monte Tabor ci mostra non soltanto l'aspetto glorioso della vita di Gesù, ma anche quello della sofferenza e della morte che si racchiuda nel termine esodo: „Parlavano del suo esodo che avrebbe portato a compimento a Gerusalemme” (Lc 9,31). La Trasfigurazione conferma che la gloria del Messia può essere raggiunta attraverso la croce e nel luogo che era destinato al trionfo messianico e alla instaurazione del Regno cioè a Gerusalemme ${ }^{60}$.

Questo riferimento dell'Esortazione all'esodo getta pure luce sulla dimensione pasquale della vita consacrata. Essa è profondamente unita a Cristo e destinata a condividere il cammino della croce che il Signore ha aperto. Nella vita dei consigli evangelici le persone consacrate sperimentano momenti di gioia intensa, che vengono concessi secondo un disegno di grazia che aiuta a scoprire ogni giorno la persona di Gesù e a sperimentare la verità e la gioia di un dono totale a lui e al suo Regno. Ma nella vita, che è imitazione di Gesù, non si possono eliminare i momenti di difficoltà e di sofferenza. La fedeltà ai consigli evangelici presuppone pure la perseveranza nelle prove e l'accettazione di spoliazioni. Come

${ }^{58} \mathrm{~J}$. Beyer, Vita consacrata: dottrina conciliare e sviluppi ulteriori, in: Vaticano II: Bilancio e prospettive venticinque anni dopo (1962-1987), Assisi 1988, 1125.

${ }_{59}$ Cf. A. Mistrorigo, Consigli evangelici, in: IDEM, Guida alfabetica alla vita spirituale, Casale Monferrato 1998, 82.

${ }^{60}$ Cf. J. Galot, La Trasfigurazione, op. cit., 358. 
Gesù per la sua umanità ha raggiunto il vertice nel dono della vita, così nella vita dei consacrati la croce è necessaria per farsi dono totale a $\mathrm{Dio}^{61}$.

La profonda e conveniente comprensione della dimensione pasquale della vita consacrata non porta, secondo il documento, ad uno sguardo negativo ai consigli evangelici. L'Esortazione vuole sottolineare questo nelle seguente parole: „Ai tre discepoli estasiati giunge l'appello del Padre a mettersi in ascolto di Cristo, a porre in Lui ogni fiducia, a farne il centro della vita. Nella parola che viene dall'alto acquista nuova profondità l'invito col quale Gesù stesso, all'inizio della vita pubblica, li aveva chiamati alla sua sequela, strappandoli alla loro vita ordinaria e accogliendoli nella sua intimità. Ė proprio da questa speciale grazia di intimità che scaturisce, nella vita consacrata, la possibilità e l'esigenza del dono totale di sé nella professione dei consigli evangelici" ${ }^{62}$. Alla luce di questo brano appare più chiaramente il valore positivo dei consigli evangelici e della consacrazione totale a Dio. Questo è molto importante perché spesso si pone attenzione più grande all'aspetto negativo, invece di mostrare i valori positivi della sequela di Gesù. Il documento dice che i consigli "prima e più che una rinuncia, sono una specifica accoglienza del mistero di Cristo, vissuta all'interno della Chiesa" ${ }^{63}$. Evidentemente quest'affermazione non nega la necessità della rinuncia perché essa è una componente essenziale dei consigli evangelici. Essa mostra soltanto che il fondamento essenziale di questo stato di vita è l'amore per Cristo, il quale esige anche le rinunce che permettono di prendere possesso di tutto il cuore del discepolo.

Gian Franco Poli invece osserva che non possiamo limitare la comprensione della vita consacrata soltanto ad una consacrazione a Dio realizzata tramite la rinuncia al mondo, come può verificarsi anche in religioni non cristiane. Questa forma di vita è una relazione intrinseca ed essenziale con Gesù Cristo. Le persone lo seguono non come un buon maestro di saggezza, ma come il Signore. Scegliere il modo di vita secondo i consigli evangelici è una risposta al suo invito a seguirlo. Questa
${ }^{61}$ Cf. ibid., 358-359.
62 VC 16.
$63 \mathrm{VC} 16$. 
sua chiamata è incondizionata (cf. Mc 1,$7 ; 2,14)$ e porta l'uomo nel servizio di Dio (cf. Mc 1,15). „Gli stessi consigli evangelici e la vita comunitaria sono riferiti a Gesù proprio per la loro nota di «totale disponibilità» al progetto di Dio. Lo stesso seguire in maniera «piena» è un segno per il popolo di Dio, come lo fu il gruppo dei dodici per Israele"64.

Lo sguardo positivo ai consigli evangelici aiuta di comprendere che non è un profondo distacco che identifica il radicalismo della vita consacrata, bensì la radicalità dell'appartenenza. Secondo Bruno Maggioni, mostrano questo tutti i passi evangelici dove il lasciare le cose e il seguire Gesù sono uniti indissolubilmente. Il Regno, avendo un carattere di assoluta novità, „non può essere dunque accolto senza passare attraverso un profondo distacco. Non c'è sequela senza esodo" ${ }^{65}$.

Potremmo dire che la scelta dei consigli evangelici da parte di Gesù è un elemento fondamentale del suo svuotamento. Egli realizzava la sua kenosi nella vita povera, nella spoliazione di tutto, ha voluto essere solo e incompreso, ha voluto essere servo e dipendente in tutto dal Padre. In questa ottica è chiaro che la rinuncia anche per i suoi discepoli appartiene all'essenza della vocazione legata alla professione dei consigli evangelici poiché essi in modo peculiare introducono le persone consacrate nel mistero dell'annientamento del Signore ${ }^{66}$.

La teologia postconciliare presenta i consigli evangelici come via per l'acquisizione degli autentici valori umani nella sequela di Cristo. A questo punto vediamo il bisogno d'aprirsi della persona consacrata e di ricevere questo dono gratuitamente dato ${ }^{67}$. Jean Galot pone l'accento su questa

${ }^{64}$ G. F. Poli, La teologia della vita consacrata dal Concilio ad oggi, in: La vita consacrata. Un carisma da riscoprire nella Chiesa comunione-missione, Padova 1994, 100.

65 B. MAGgioni, Il fondamento evangelico della vita consacrata, op. cit., 108. Lautore sviluppa questo tema servendosi dei diversi brani del Vangelo. Cf. ibid., 108-112. Sul tema del radicalismo: T. MATURA, Il radicalismo evangelico, Roma 1981; B. BAUR, Alle origini del radicalismo evangelico, Brescia 1983.

${ }^{66}$ Cf. RD 10; A. Pigna, Il dono dei consigli evangelici, „Rivista di Vita Spirituale” 54 (2000), 175-176.

${ }^{67}$ Cf. P. G. CABra, I consigli evangelici un dono per la missione, op. cit., 592; A. PIGNA, Fondamenti teologici della vita consacrata, „Rivista di Vita Spirituale” 51 (1997), 88-89; IDEM, Il dono dei consigli evangelici, op. cit., 173-174. 
comprensione positiva della vita consacrata: „L'episodio della Trasfigurazione fa capire che un autentico entusiasmo per la persona di Cristo, è l'anima della vita consacrata. I primi discepoli che hanno seguito Cristo, hanno lasciato volentieri tutto per poter vivere per lui e con lui. Nel racconto delle prime vocazioni, come vengono riportate nel Vangelo di Giovanni, viene messo in evidenza l'entusiasmo per aver trovato il Messia. Questo entusiasmo dell'inizio non si è mai spento. La Trasfigurazione lo risveglia e lo conferma, in vista della prova della passione. Non si tratta di un entusiasmo provocato da semplici motivi sentimentali. La persona di Cristo si rivela come persona divina, attraverso una vita umana. Come persona divina, egli esercita un'ttrazione molto potente e intima. Suscita un'adesione assoluta di fede e di amore. Il senso di questa attrazione viene chiarito dalla voce del Padre quando proclama Gesù, Figlio suo prediletto" ${ }^{\prime 68}$. Vediamo così che il motore principale della vita consacrata è un autentico entusiasmo per Gesù che permette di lasciare tutto per lui. Mostrano questo in modo molto chiaro i brani evangelici che descrivono la vocazione dei primi discepoli di Gesù. I consigli evangelici, per l'imitazione di vita di Gesù, portano le persone consacrate alla pienezza e all'anticipazione nel tempo di vita eterna. „La consacrazione meglio preannunzia la futura risurrezione e la gloria del Regno celeste. Questo fa innanzitutto la scelta verginale, sempre intesa dalla tradizione come un'anticipazione del mondo definitivo, che già fin da ora opera e trasforma l'uomo nella sua interezza" ${ }^{69}$.

L'Esortazione Vita Consecrata non per caso usa l'icona del Cristo trasfigurato. Questo evento è importante per i tutti consacrati. Come gli apostoli sono stati fortificati e preparati alla prova della croce, così i consacrati sono rinforzati nella loro vita di rinuncia e di imitazione di Gesù. Gesù nella Trasfigurazione dimostra ai suoi discepoli che avevano fatto bene a legare la loro vita alla sua. La vita consacrata in qualche senso è unanticipazione della risurrezione, dello stato glorioso. Rinunciando allamore umano del matrimonio, i religiosi si impegnano nella via dei consigli evangelici e vogliono formare un'unione più intima e più imme-

\footnotetext{
68 J. Galot, La Trasfigurazione, op. cit., 360.

${ }^{69}$ VC 26. Cf. LG 44.
} 
diata con Gesù, la quale costituisce un primo anticipo dell'unione celeste. Anche imitare il Cristo nella povertà rinunciando al possesso dei beni terreni per cercare lui come il suo tesoro unico, è un stato di annunciare il possesso definitivo nella vita eterna. I religiosi non hanno ricevuto una visione speciale di Cristo trasfigurato, ma nella luce della Trasfigurazione possono scoprire la presenza profonda e vera di Cristo glorioso nella loro vita. „Essi devono credere allo splendore di colui che vive segretamente nel loro cuore, devono essere sempre più consapevoli della grandezza di una vita che appare spesso del tutto ordinaria, ma che è arricchita dalla presenza sovrana di Cristo" 70 .

Potremo dire che la sequela di Cristo nella via dei consigli evangelici mostra Gesù come il valore supremo delle persone consacrate. Come dice Paolo: „Anzi, tutto ormai io reputo una perdita di fronte alla sublimità della conoscenza di Gesù Cristo, mio Signore, per il quale ho lasciato perdere tutte queste cose e le considero come spazzatura, al fine di guadagnare Cristo e di essere trovato in lui" (Fil 3,8-9). La scelta dei valori che si mostrano nei consigli evangelici è in fondo la scelta di Gesù, Servo di Dio ${ }^{71}$.

Assumendo il tema della dimensione pasquale della vita consacrata che ci propone l'Esortazione possiamo dire che essa si esprime anzitutto nei tre livelli: nel battesimo, nell'Eucaristia e nei consigli evangelici. Come abbiamo mostrato, sia il battesimo sia l'Eucaristia sono elementi comuni di tutta la vita cristiana, dunque sono offerti a tutti cristiani per approfondire la loro unità con Gesù. I cristiani, attraverso il battesimo, entrano nella morte di Cristo per risorgere con lui nella risurrezione. Qui si comincia lo stretto legame con il Signore, il quale si sviluppa poi nell'Eucaristia. Essa è il mistero pasquale per eccellenza e il centro di vita di ogni seguace di Cristo. I consigli evangelici invece sono la via più stretta sulla quale i consacrati seguono Gesù imitando il suo modo di vita. In questa nuova e speciale consacrazione a Dio sta la novità della vita consacrata rispetto alla vita gli altri battezzati.

\footnotetext{
70 J. Galot, La Trasfigurazione, op. cit., 356.

${ }^{71}$ Cf. A. Pigna, Il dono dei consigli evangelici, op. cit., 177.
} 


\section{Testimoni di Cristo nel mondo odierno}

Ogni dono di Dio non è mai concesso solo per suscitare ammirazione. Il dono genera sempre una responsabilità. Così le persone consacrate, conoscendo la grandezza di questa donazione divina ricevuta specialmente nei consigli evangelici, per la loro vita diventano vera testimonianza di Dio, di cui sono imitatori in modo speciale. La testimonianza di Cristo nella vita dei consacrati si orienta intorno alla consacrazione a Dio attraverso i consigli evangelici e alla missione ${ }^{72}$.

Elemento forte di testimonianza di Gesù è senza dubbio la vita stessa dei consacrati, che vogliono imitare Gesù nella castità, povertà ed obbedienza. I consigli evangelici diventano in tal modo un segno del servizio a Gesù e al Regno di Dio ${ }^{73}$. In questo contesto emerge anche il carattere cristologico e trinitario dei consigli evangelici, che sono "una speciale espressione dell'amore che il Figlio porta al Padre nell'unità dello Spirito Santo"74. L'Esortazione sottolinea pure il loro profondo significato antropologico: „La scelta di questi consigli, infatti, lungi dal costituire un impoverimento di valori autenticamente umani, si propone piuttosto come una loro trasfigurazione. I consigli evangelici non vanno considerati come una negazione dei valori inerenti alla sessualità, al legittimo desiderio di disporre di beni materiali e di decidere autonomamente di sé. Queste inclinazioni, in quanto fondate nella natura, sono in se stesse buone. (...) La professione di castità, povertà e obbedienza diventa monito a non sottovalutare le ferite prodotte dal peccato originale e, pur affermando il valore dei beni creati, li relativizza additando Dio come il bene assoluto"75.

Abbracciando la verginità, come Gesù, i consacrati vogliono offrire il loro amore al Padre. Imitando la sua povertà confessano che il Figlio tutto riceve dal Padre e nell'amore tutto gli restituisce (cf. Gv 17,7.10). Per il sacrificio della propria libertà lo confessano „,come Colui che si com-

72 Cf. J. GAlot, Cristo fondatore della vita consacrata, op. cit., 311-312; B. SECONDIN, Guardando al futuro, in: Vita Consecrata. Una prima lettura teologica, op. cit., 176-177.

73 Cf. VC 22.

74 G. F. Ghirlanda, L'esortazione apostolica Vita Consecrata continua a provocare i consacrati, „Vita Consacrata” 2(34) (1998), 146.

${ }^{75} \mathrm{VC} 87$. 
piace solo della volontà del Padre (cf. Gv 4,34), al quale è perfettamente unito e dal quale in tutto dipende" ${ }^{\text {" }}$. Tale stretta e conformativa immedesimazione al mistero del Salvatore fa dele persone consacrate in modo speciale la viva testimonianza della „confessio Trinitatis che caratterizza l'intera vita cristiana, riconoscendo con ammirazione la sublime bellezza di Dio Padre, Figlio e Spirito Santo e testimoniandone con gioia l'amorevole condiscendenza verso ogni essere umano"77.

Di fronte alla provocazione della cultura contemporanea che si caratterizza di una tendenza edonistica e narcisistica, le persone consacrate scelgono la pratica gioiosa della castità perfetta, la quale diventa per mezzo della loro vita una testimonianza significativa dell'amore di Dio nella fragilità della condizione umana. „La persona consacrata attesa che quanto è creduto impossibile dai più diventa, con la grazia del Signore Gesù, possibile e autenticamente liberante. Sì, in Cristo è possibile amare Dio con tutto il cuore, ponendolo al di sopra di ogni altro amore, ed amare così, con libertà di Dio, ogni creatura! Ė questa una testimonianza oggi più che mai necessaria, proprio perché così poco compresa dal nostro mondo"78.

La castità perfetta, come anche l'amore umano sponsale nel caso del matrimonio, essendo un contenuto significativo della vita consacrata, affonda la sua radice ultima e piena nellamore fecondo e gratuito di Dio Padre. Radicandosi in questo amore divino, essa afferma in modo profetico la possibilità dell'amore altruistico e crea una nuova cultura del rispetto della persona umana e della sua più profonda sacralità ${ }^{79}$. La castità non mortifica la libertà della persona consacrata, ma la esalta. D’altra parte, essa sconfigge l'idolatria dell' istinto che si esprime nel mondo d'oggi nell'uso strumentale dell'uomo da parte di un altro uomo o della stes-

${ }^{76} \mathrm{VC} 16$.

$77 \mathrm{VC} 16$.

78 VC 88. Cf. G. Calabrese, Una profezia per la Chiesa e per il mondo, „Vita Consacrata" 6(32) (1996), 608-609; G. SAVAGNONE, Il sale e la luce. Il contributo specifico della vita consacrata al progetto culturale, in: Conferenza Italiana Superiori Maggiori (red.), Consacrati da Dio, dono alla Chiesa e al mondo..., op. cit., 328-331.

79 Cf. G. F. GHIRLAnda, L'esortazione apostolica Vita Consecrata continua a provocare i consacrati, op. cit., 152-153. 
sa società. In questa luce vediamo per quale motivo il consumismo non può comprendere la castità per il Regno di Dio, che è una scelta gioiosa nella forza dello Spirito Santo e senza guardare al proprio guadagno. L'Esortazione sottolinea che la vita consacrata deve presentare al mondo odierno „esempi di una castità vissuta da uomini e donne che dimostrano equilibrio, dominio di sé, intraprendenza, maturità psicologica ed affettiva" ${ }^{30}$. Questo equilibrio e maturità delle persone consacrate nascono dalla contemplazione dell'amore trinitario che è stato rivelato in modo più pieno in Gesù Cristo. I consacrati, capaci di un amore radicale e universale, diventano così i testimoni dell'amore verso Dio e verso gli uomini, che appare come esperienza di gioia e di libertà ${ }^{81}$.

La povertà evangelica è un valore in sé, perché imita Cristo povero il quale si consacrò totalmente al Padre. Essa richiama anche la prima delle Beatitudini: „Beati voi poveri, perché vostro è il Regno di Dio” (Lc 6,20). Il primo senso della povertà evangelica è testimoniare Dio come la vera ricchezza del cuore umano. Questo è anche lo scopo dell'imitazione di Cristo povero nella vita consacrata. Nel senso più profondo, la povertà "contesta - nello stesso tempo - l'idolatria di mammona, proponendosi come appello profetico nei confronti di una società che, in tante parti del mondo benestante, rischia di perdere il senso della misura e il significato stesso delle $\operatorname{cose}^{\prime \prime 2}$. Un valore della povertà consiste in questo, che essa libera il cuore da ciò che impedisce di usare le cose secondo il loro fine, aiuta ad usarle in modo corretto, tanto quanto esse servono al fine di costruire il Regno di Dio ${ }^{83}$.

Nel mondo dove possiamo osservare una specie di svalutazione della realtà, che si riduce spesso ad oggetto da conquistare, usare e gettare via, la povertà costruisce una resistenza profonda, in nome di una rinnovata percezione del valore della natura. La testimonianza della povertà aiuta a capire che non si può ridurre la natura a oggetto da manipolare

\footnotetext{
${ }^{80}$ VC 88. Cf. PC 12.

81 Cf. G. Calabrese, Una profezia per la Chiesa e per il mondo, op. cit., 610.

${ }^{82} \mathrm{VC} 90$.

83 Cf. G. F. Ghirlanda, L'esortazione apostolica Vita Consecrata continua a provoca-
} re i consacrati, op. cit., 153. 
e dominare senza freni. D’altra parte, la povertà ricostruisce la percezione che l'uomo ha di se stesso. Nella sua ottica, l'uomo vale per quello che è e non per quello che ha. Il valore dell'uomo non dipende dal possesso o dal prestigio sociale, bensì dalla sua propria personalità ${ }^{84}$.

La testimonianza della povertà evangelica non ha soltanto un valore ascetico ma anche ecclesiologico e sociale. Essa aiuta nella liberazione dalla mentalità del possesso, dall'idolatria dell'avere e far maturare uno stile di vita nuovo e più umano di fraternità e della realizzazione del bene comune. Tale atmosfera è necessaria per la crescita e il rispetto della dignità e della sacralità della persona umana ${ }^{85}$.

Vediamo dunque che il rinnovato e vigoroso stile di vita evangelica nell'abnegazione e nella sobrietà, diventa non soltanto una testimonianza di semplicità, ma anche un forte esempio per quanti rimangono indifferenti di fronte alle necessità e alle sofferenze del prossimo. L'azione stessa dei consacrati in modo naturale si rivolge all'amore preferenziale per i poveri e deve manifestarsi pure nella condivisione delle condizioni di vita dei più desiderati ${ }^{86}$. Il documento tratta questo tema in modo seguente: „Non sono poche le comunità che vivono e operano tra i poveri e gli emarginati, ne abbracciano la condizione e ne condividono le sofferenze, i problemi e i pericoli. Grandi pagine di storia di solidarietà evangelica e di dedizione eroica sono state scritte da persone consacrate, in questi anni di profondi cambiamenti e di grandi ingiustizie, di speranze e di delusioni, di importanti conquiste e di amare sconfitte. E pagine non meno significative sono state e sono tuttora scritte da altre innumerevoli persone consacrate, le quali vivono in pienezza la loro vita «nascosta

${ }^{84}$ Cf. G. Savagnone, Il sale e la luce..., $d z$. cyt., 331-333.

${ }^{85}$ Cf. G. Calabrese, Una profezia per la Chiesa e per il mondo, op. cit., 612.

${ }^{86}$ Cf. VC 82; G. Calabrese, Una profezia per la Chiesa e per il mondo, op. cit., 619-621; L. CRIPPA, Opzione preferenziale per i poveri, in: Dizionario Teologico della Vita Consacrata, op. cit., 1174-1187; U. SARTORIO, La testimonianza carismatica dei consacrati nella Chiesa e di fronte al mondo, in: La vita consacrata. Un carisma da riscoprire nella Chiesa comunione-missione, op. cit., 256-262; G. VALENTINI, L'opzione preferenziale per i poveri è di tutti, ma soprattutto dei consacrati, in: P. Vanzan, F. Volpi (red.), Il giubileo e la vita consacrata..., op. cit., 165-173; F. ZАмвотті, Le nuove povertà interpellano la vita consacrata, in: P. Vanzan, F. Volpi (red.), Il giubileo e la vita consacrata..., op. cit., 175-180. 
con Cristo in Dio» (Col 3,3) per la salvezza del mondo, allinsegna della gratuità, dell'investimento della propria vita in cause poco riconosciute e meno ancora applaudite. Attraverso queste forme diverse e complementari, la vita consacrata partecipa all'estrema povertà abbracciata dal Signore e vive il suo specifico ruolo nel mistero salvifico della sua incarnazione e della sua morte redentrice" ${ }^{87}$.

La povertà, che scelgono le persone consacrate, appare come risposta alla concezione materialistica della vita e alla legge del possesso. Essa è spesso unita con una azione nella promozione della solidarietà e della carità. In questa luce possiamo vedere chiaramente che la povertà evangelica possiede il carattere fortemente antropologico, che non solo si distingue per la sua natura ascetica e spirituale, ma anche per la sua rilevanza sociale ${ }^{88}$.

Nella storia della Chiesa vediamo una molteplicità di istituzioni religiose e di persone consacrate che vivono il consiglio evangelico della povertà e hanno offerto la loro vita per la difesa delle legittime esigenze di emancipazione sociale, economica e culturale. L'Esortazione elenca anche gli istituti dedicati all'educazione, all'istruzione, alla formazione professionale ed aggiunge i consacrati che si spendono per gli ultimi della terra, per la formazione di futuri educatori e responsabili della vita sociale ${ }^{89}$.

Riconoscendo e contribuendo a rimediare alle esigenze del mondo doggi, i consacrati per la loro consacrazione nella povertà diventano i veri operatori di carità, portatori dell'amore e testimoni del Cristo povero. Per le diverse e complementari forme di vita e di azione, la vita consacrata partecipa in modo molto profondo all'estrema povertà del Signore e al suo mistero salvifico ${ }^{90}$.

Le persone consacrate, per la libera e responsabile scelta della obbedienza evangelica, rivelano Dio come sorgente del bene dell'uomo. Facendosi simili a Cristo nella sua obbedienza al Padre, essi confessano il primato di Dio per la vita e la realizzazione delluomo. Questa

\footnotetext{
87 VC 90.

${ }^{88}$ Cf. VC 89; G. Calabrese, Una profezia per la Chiesa e per il mondo, op. cit., 611.

89 Cf. VC 89.

90 Cf. VC 90.
} 
testimonianza dell'obbedienza esprime anche la loro figliolanza, la quale - come Cristo - assume la volontà del Padre come cibo quotidiano (cf. Gv 4,34). La vera obbedienza al Signore conduce a vivere nella piena libertà che non porta alla distruzione o negazione, ma a vivere nella verità nel rapporto con il cosmo e con Dio stesso ${ }^{91}$. L'obbedienza „ripropone in modo particolarmente vivo l'obbedienza di Cristo al Padre e, proprio partendo dal suo mistero, testimonia che non cè contraddizione tra obbedienza e libertà. In effetti, l'atteggiamento del Figlio svela il mistero della libertà umana come cammino d’obbedienza alla volontà del Padre e il mistero dellobbedienza come cammino di progressiva conquista della vera libertà"92. La persona consacrata, assumendo il consiglio dell'obbedienza evangelica, attesta quindi il suo rapporto di figliolanza con Dio e cresce nella piena libertà di se stessa, rimanendo collegata con la fonte della sua esistenza ${ }^{93}$.

Anche la dimensione comunitaria dell'obbedienza alla volontà del Padre, diventa un segno di fronte alla Chiesa e alla società, perché tutti sono chiamati a compiere la volontà di Dio. In questo senso, lobbedienza evangelica, contro lo spirito di discordia e di divisione, risplenda come un segno dell'unità paterna che viene da Dio, della fraternità di tutti gli uomini nata dallo Spirito e della figliolanza mutua rivelata in Gesù Cristo $^{94}$. Questo compiere insieme la volontà del Padre senza dubbio è un segno forte dell'unità e fa efficace la missione dei consacrati nel mondo. Il Papa scrive: „L'obbedienza, vivificata dalla carità, unifica i membri di un istituto nella medesima testimonianza e nella medesima missione, pur nella diversità dei doni e nel rispetto delle singole individualità. (...) Inoltre, chi obbedisce ha la garanzia di essere davvero in missione, alla sequela del Signore e non alla rincorsa dei propri desideri o delle proprie aspettative. E così è possibile sapersi condotti dallo Spirito del

91 Cf. G. Calabrese, Una profezia per la Chiesa e per il mondo, op. cit., 613-616.

92 VC 91.

93 Cf. G. F. GHiRlanda, Lesortazione apostolica Vita Consecrata continua a provocare i consacrati, op. cit., 154.

94 Cf. PC 14: „L’obbedienza religiosa, anziché diminuire la dignità della persona umana, la conduce a maturità, facendo crescere la libertà dei figli di Dio". 
Signore e sostenuti, anche in mezzo a grandi difficoltà, dalla sua mano sicura (cf. At 20,22-23)" ${ }^{\prime 95}$.

Nel odierna crisi dell'autorità, la testimonianza dei consacrati aiuta capire meglio che senza obbedienza non c’è libertà e viceversa. L'autonomia assoluta dell'individuo, come equivalente della libertà, porta l'uomo alla solitudine ed alla contraddizione. G. Savagnone afferma che „spetta alla vita consacrata l'impegnativo compito di testimoniare la possibilità di conciliare libertà e obbedienza e di ispirare una cultura dove questi due poli, oggi sentiti come antitetici, rivelano la loro necessaria complementarietà" 96 .

Lo scopo della sequela di Cristo e di condividerne la vita è la missione. Le persone consacrate perciò sono chiamate a dedicarsi totalmente alla missione. Il documento afferma anche che tutta la vita consacrata, come imitazione di vita di Gesù, è una missione speciale, la quale annuncia e rende presente Cristo nel mondo. Il fonte della missione è la Trinità dove il Padre personifica il primato dell'iniziativa divina. Il Papa scrive: „Ad immagine di Gesù, Figlio diletto «che il Padre ha consacrato e mandato nel mondo» (Gv 10,36), anche coloro che Dio chiama alla sua sequela sono consacrati ed inviati nel mondo per imitarne l'esempio e continuarne la missione. Fondamentalmente, questo vale per ogni discepolo. In modo speciale, tuttavia, vale per quanti, nella forma caratteristica della vita consacrata, sono chiamati a seguire Cristo «più da vicino», e a fare di Lui il «tutto» della loro esistenza" ${ }^{\text {"97. }}$

Per il loro modo di vita aperto alla missione, che è natura stessa della vita consacrata, le persone consacrate si collocano nella natura stessa della Chiesa, in una dimensione teologica, escatologica e missionaria. Questa natura missionaria porta ad un rapporto di ministerialità con il mondo contemporaneo, ad unazione di civilizzazione, di santi-

95 VC 92. Cf. G. F. GHirlanda, Lesortazione apostolica Vita Consecrata continua a provocare i consacrati, op. cit., 154-155.

96 Cf. G. SAvagnone, Il sale e la luce..., dz. cyt., 333.

97 VC 72. Cf. VC 17; G. F. GHIRLANDA, Lesortazione apostolica Vita Consecrata continua a provocare i consacrati, op. cit., 140-141; S. GonzÁLES SILva, Consacrati e inviati, in: Vita Consecrata. Una prima lettura teologica, op. cit., 110-112. 
ficazione, di inculturazione della fede e di promozione umana e sociale nel mondo ${ }^{98}$.

La missione dei consacrati si traduce nella testimonianza della propria vita di consacrazione, nella generosità del servizio nella Chiesa secondo il proprio carisma e nella stessa vita fraterna in comunità ${ }^{99}$. L'Esortazione rivolge l'attenzione al fatto che "la missione è essenziale per ogni istituto, non solo in quelli di vita apostolica attiva, ma anche in quelli di vita contemplativa" ${ }^{100}$. Al primo posto il documento elenca la testimonianza personale dei consacrati, che deve sempre precedere tutte le opere esteriori. Essa è nominata come il compito primario della vita consacrata. „Più ci si lascia conformare a Cristo, più lo si rende presente e operante nel mondo per la salvezza degli uomini"101. G. F. Ghirlanda chiama questo tipo di missione la "missione dellessere" e non innanzitutto „del fare”. Nella vita quotidiana secondo il proprio carisma essa diventa una vera missione di testimonianza, che si esprime nella vita individuale e collettiva, nella propria fedeltà. Tale testimonianza diventa una amorosa risposta al dono della consacrazione ricevuta dal Signore ed affermazione della trascendenza dell'amore del Padre ${ }^{102}$.

La testimonianza di vita, secondo il proprio carisma, ed le opere di apostolato e di promozione umana compiute nella Chiesa sono ugualmente necessarie perché raffigurano Cristo che è venuto come consacrato al Padre ed inviato per la salvezza dei suoi fratelli e delle sue sorelle ${ }^{103}$.

Ė interessante, che l'Esortazione sottolinea che la comunità religiosa è spazio teologale in qui si può sperimentare la presenza del Signore risorto. Solamente dopo, essa è lo strumento per una determinata missione. La viva presenza di Gesù è sperimentata grazie all'amore reciproco dei fratelli, un amore alimentato dalla Parola e dall'Eucaristia, purificato nel Sacramento della Riconciliazione, sostenuto dalla vivificante azione

${ }^{98}$ Cf. G. Calabrese, Una profezia per la Chiesa e per il mondo, op. cit., 602-603.
99 Cf. A. Pigna, I contenuti, op. cit., 304.
100 VC 72 a.
101 VC 72 b.
102 Cf. G. F. GHirlanda, Lesortazione apostolica Vita Consecrata continua a provocare $i$ consacrati, op. cit., 142.

${ }^{103}$ Cf. VC 72; LG 46. 
dello Spirito Santo - donatore dell'unità ${ }^{104}$. Ugualmente le costituzioni dei molti istituti mettono in risalto l'importanza della comunità nel testimoniare Cristo. Come esempio prendiamo qui il brano dalle Costituzione dei Sacerdoti del Sacro Cuore di Gesù: „Nella comunione, anche al di là dei conflitti, e nel perdono vicendevole, vorremo testimoniare che la fraternità di cui gli uomini hanno sete è possibile in Gesù Cristo e noi vorremo esserne i servitori. La comunità si sforza di testimoniare Cristo nel cui nome è riunita. Nello stesso tempo essa può portare un aiuto prezioso alla crescita delle persone che la compongono"105.

Pure la fraternità dei consacrati nelle relazione con la Chiesa universale e locale è la testimonianza dell'unità della Trinità. L'Esortazione parla del peculiare vincolo di comunione che le varie forme di vita consacrata hanno sempre avuto con il successore di Pietro. Lo Spirito di comunione e collaborazione con le diverse Chiese particolari è anche un elemento valido di testimonianza ${ }^{106}$.

Il documento pone l'attenzione parimenti sulla collaborazione con i laici. Essi sono invitati a condividere e partecipare in modo più intenso alla spiritualità e alla missione dei diversi istituti. Questa profonda comunione conduce alla crescita spirituale sia dei laici, sia dei consacrati, stimola lo sviluppo della missione e l'arricchimento stesso del carisma e della vita propria dei consacrati ${ }^{107}$. Il Papa afferma: „Oggi non pochi istituti, spesso in forza delle nuove situazioni, sono pervenuti alla convinzione che il loro carisma può essere condiviso con i laici. Questi vengono perciò invitati a partecipare in modo più intenso alla spiritualità e alla missione dell'istituto medesimo. Si può dire che, sulla scia di esperienze storiche come quella dei diversi Ordini secolari o Terz’Ordini, è iniziato un nuovo capitolo, ricco di speranze, nella storia delle relazioni tra le persone consacrate e il laicato"108.

104 Cf. VC 42; A. Pigna, I contenuti, op. cit., 299.

105 Congregazione dei Sacerdoti del Sacro Cuore di Gesù (Dehoniani), Costituzioni, Milano-Napoli 1987, 65-67.

106 Cf. VC 47; A. Pigna, I contenuti, op. cit., 300.

107 Cf. VC 55.

108 VC 54. 
Nel numero 16 l'Esortazione mostra i compiti dei tre stati di vita: dei laici, dei ministri sacri e dalla vita consacrata. Il compito affidato ai consacrati è „,additare il Figlio di Dio fatto uomo come il traguardo escatologico a cui tutto tende, lo splendore di fronte al quale ogni altra luce impallidisce, l'infinita bellezza che, sola, può appagare totalmente il cuore dell'uomo"109. In questo compito si sente non soltanto la chiamata di Gesù all'amore totale, „più del padre e della madre, più del figlio o della figlia" (cf. Mt 10,37) come ha chiesto ai suoi discepoli. I consacrati devono esprimere con la loro vita una tensione totalizzante al Cristo che anticipa, in qualche modo, la perfezione escatologica ${ }^{110}$.

Anche nel campo della cultura le persone consacrate, attraverso la speciale forma di vita, provocano la coscienza degli uomini diventando pure un fermento evangelico capace di purificare e fare evolvere la cultura odierna. Il Papa sottolinea questo nel numero 80 dell'Esortazione: „Le comunità degli istituti religiosi e delle Società di vita apostolica possono, infatti, offrire concrete e significative proposte culturali, quando testimoniano il modo evangelico di vivere l'accoglienza reciproca nella diversità e di esercitare lautorità, la condivisione dei beni sia materiali che spirituali, l'internazionalità, la collaborazione inter-congregazionale, lascolto degli uomini e delle donne del nostro tempo. Il modo di pensare e di agire di chi segue Cristo più da vicino, infatti, dà origine ad una vera e propria cultura di riferimento, serve a mettere in luce ciò che è disumano, testimonia che Dio solo dà ai valori forza e compimento" 111 .

L’impegno peculiare dei consacrati è di avere il coraggio di ridefinire l'identità ultima dell'uomo. Il documento pone l'accento sul fatto che gli istituti di vita consacrata lungo la storia hanno sempre avuto un gran-

109 VC 16. Cf. G. L. Pussino, Laici e persone consacrate: quali rapporti?, in: Conferenza Italiana Superiori Maggiori (red.), Consacrati da Dio, dono alla Chiesa e al mondo... op. cit., 340-356.

110 Sulla relazione tra i consacrati e laici vedi anche: A. BARRUfFo, Laico, in: S. De Fiores, T. Goffi (red.), Nuovo dizionario di spiritualità, op. cit., 823-825; L. GuccINI, Religiosi e laici insieme verso il domani, in: Una comunità per domani. Prospettive della vita religiosa apostolica, Bologna 2000, 57-79.

111 VC 80. Cf. G. Calabrese, Una profezia per la Chiesa e per il mondo, op. cit., 608. 
de influsso nella formazione e nella trasmissione della cultura. La prima parte del punto 98, dedicato all'evangelizzazione della cultura, fa ricordare questo influsso con l'esempio di diversi campi della cultura. Nella parte seguente il Papa invita i consacrati a tradurre la fedeltà ai principi di fondo, validi per ogni tempo ed ogni luogo, in un linguaggio nuovo, accessibile agli uomini e alle donne d'oggi. „I consacrati non possono non sentirsi interpellati da questa urgenza. Anchessi sono chiamati a individuare nell'annuncio della Parola di Dio, metodi più appropriati alle esigenze dei diversi gruppi umani e dei molteplici ambiti professionali, perché la luce di Cristo penetri ogni settore umano ed il fermento della salvezza trasformi dall'interno il vivere sociale, favorendo l'affermarsi di una cultura permeata di valori evangelici" ${ }^{\prime 12}$.

La missione nel mondo della cultura non si limita soltanto al servizio rivolto agli altri. Il documento pone in rilievo anche un bisogno di dedizione allo studio come mezzo della formazione integrale dell'uomo e anche come percorso ascetico. Lo studio non fa parte soltanto della formazione iniziale alla vita religiosa e non si può ridurlo al conseguimento di titoli accademici e di competenze professionali. Tutto lo sforzo umano in questa materia serve ad approfondire la conoscenza di Dio. Per questo, dice il documento, „tale impegno non isola la persona consacrata in un astratto intellettualismo, né la rinchiude nelle spire di un soffocante narcisismo; è invece sprone al dialogo e alla condivisione, è formazione alla capacità di giudizio, è stimolo della contemplazione e alla preghiera, nella continua ricerca di Dio e della sua azione nella complessa realtà del mondo contemporaneo" ${ }^{113}$. Possiamo, quindi, dire che levangelizzazione della cultura porta le persone consacrate ad una più intensa e profonda esperienza di fede dalla quale, nell'accordo con le parole di G. Savagnone, nasce una nuova alleanza tra Vangelo e cultura, tra esperienza spirituale e vita intellettuale, tra contemplazione delle cose divine e sforzo umano ${ }^{114}$.

112 VC 98b. Cf. G. SAVAGNONE, Il sale e la luce..., dz. cyt., 316-320.

${ }_{113}$ VC 98c.

${ }^{114}$ Cf. G. Savagnone, Il sale e la luce..., $d z$. cyt., 323. 
Nel mondo d'oggi si aprono davanti alla vita consacrata le grandi sfide e gli ampi orizzonti della testimonianza sul Cristo. Per essere in grado di venire incontro ad essi i consacrati devono raggiungere una profonda esperienza di Dio e allo stesso tempo aprirsi agli interiori suggerimenti dello Spirito Santo, il quale invita e chiama „a elaborare nuove risposte per i nuovi problemi del mondo di oggi" 115 . In questo modo possono nascere nuovi progetti di evangelizzazione e nuove opere che saranno una risposta ai segni del tempo ${ }^{116}$.

Ogni opera ed ogni azione dei consacrati deve partire dallamore con il quale Gesù ci amò sino alla fine. Nella lavanda dei piedi il Signore ci rivela non soltanto la profondità dell'amore di Dio per l'uomo, ma anche il modo d'agire e di comportarsi. Il Papa osserva che „Egli rivela, al tempo stesso, il senso della vita cristiana e, a maggior ragione, della vita consacrata, che è vita d'amore oblativo, di concreto e generoso servizio" 117 .

La missione e la testimonianza di Cristo esige dai consacrati una fedeltà alla vocazione come dono divino e soltanto così può portare i frutti per gli altri e per loro stessi. „Oggi come mai la scelta dei religiosi può apparire estranea e inattuale, rispetto alle urgenze del mondo contemporaneo. Ma proprio questa apparente estraneità rivela la potenziale novità del messaggio che essi possono offrire agli uomini e alle donne del nostro tempo. E così, come il sale e la luce, restando fedeli a quel che sono, saranno anche in grado di aiutare i loro fratelli a riscoprire il gusto e la gioia della vita" ${ }^{\prime 118}$.

\section{Conclusione}

Nel questo articolo volevo sottolineare la dimensione cristologica della vita consacrata. Non ci può essere nessun altro modello più chiaro, per le persone consacrate, come lo è Gesù Cristo durante la sua vita terrena.

\footnotetext{
$115 \mathrm{VC} 73$.

116 Cf. VC 73; A. Pigna, I contenuti, op. cit., 304.

117 VC 75.

118 G. Savagnone, Il sale e la luce..., dz. cyt., 336.
} 
Lui stesso, come primo consacrato, ha aperto la nuova strada attraverso i consigli evangelici, i quali si mostrano come tratti caratteristici di Gesù in questa nuova forma di vita.

Come la consacrazione di Gesù, così la consacrazione dei suoi seguaci è legata in modo molto stretto alla missione, cioè l'annuncio del Padre e il compimento della sua volontà. In questa prospettiva avevo mostrato la dimensione pasquale della vita consacrata concentrandomi prima di tutto sul battesimo, sull'Eucaristia e sui consigli evangelici. Questi tre elementi in modo peculiare rivelano la dimensione pasquale della vita consacrata.

Lo scopo della vita delle persone consacrate non è soltanto la propria santificazione. Dal fatto della sequela di Cristo emerge anche la testimonianza che aiuta agli altri suoi discepoli a scoprire la ricchezza della persona di Gesù e a prendere la decisione di camminare sui suoi passi. La testimonianza di Cristo è soprattutto la vita stessa delle persone consacrate, che essendo fedeli alla loro vocazione ed ai consigli evangelici, svolgono nella Chiesa la loro missione di annunciare Cristo come unico Salvatore del mondo. Nel clima dell'amore mutuo delle comunità religiose e nella collaborazione con i laici si colma la preghiera di Gesù „perché tutti siano una sola cosa, (...) perché il mondo creda che tu mi hai mandato" (Gv 17,21). La rivelazione di Cristo nel mondo, anche attraverso la evangelizzazione della cultura, ristabilisce la vera dignità dell'uomo e delle cose create.

\section{Bibliografia}

Amato A., Il volto di Gesù Cristo, il consacrato e il missionario del Padre nella vita consacrata, in: Conferenza Italiana Superiori Maggiori (red.), Consacrati da Dio, dono alla Chiesa e al mondo. Approfondimenti sull'Esortazione «Vita Consecrata», Roma 1997, 147-166.

Amato A., Spunti di lettura cristologico-trinitaria della Vita Consecrata, in: Vita Consecrata. Una prima lettura teologica, Milano 1996, 23-54.

Bandera A., Magistero postconciliare, in: Dizionario Teologico della Vita Consacrata, Milano 1994, 917-935. 
Bandera A., Un silenzio e un clamore, „Vita Consacrata” 3-4(32) (1996), 312-338.

Bandera A., Vita Consecrata: Il numero iniziale, „Vita Consacrata” 3(33) (1997), 244-264.

Barruffo A., Laico, in: S. De Fiores, T. Goffi (red.), Nuovo dizionario di spiritualità, Cinisello Balsamo (MI) 1999, 810-828.

Baur B., Alle origini del radicalismo evangelico, Brescia 1983.

Beyer J., Vita consacrata: dottrina conciliare e sviluppi ulteriori, in: Vaticano II: Bilancio e prospettive venticinque anni dopo (1962-1987), Assisi 1988, 1119-1139.

Cabra P. G., I consigli evangelici un dono per la missione, „Vita Consacrata” 6(32) (1996), 588-601.

Cabra P. G., La missione nella Esortazione apostolica, in: Vita Consecrata. Studi e riflessioni, Roma 1996, 187-213.

Cabra P. G., Un itinerario spirituale a partire dalle icone, in: Conferenza Italiana Superiori Maggiori (red.), Consacrati da Dio, dono alla Chiesa e al mondo. Approfondimenti sull'Esortazione "Vita Consecrata», Roma 1997, 115-145.

Calabrese G., Una profezia per la Chiesa e per il mondo, „Vita Consacrata” 6(32) (1996), 602-635.

Castellano Cervera J., Battesimo, in: Dizionario enciclopedico di spiritualità, Roma 1995, 278-290.

Castellano Cervera J., Dimensione teologica e spirituale della vita consacrata: tradizione, novità, profezia, in: Vita Consecrata. Studi e riflessioni, Roma 1996, 35-67.

Castellano Cervera J., Eucaristia, in: Dizionario enciclopedico di spiritualità, Roma 1995, 956-974.

Castellano Cervera J., Il mistero e la missione di Cristo nella vita consacrata, in: „Vita Consacrata” 3-4(32) (1996), 340-350.

Ciardi F., Il radicamento evangelico della vita consacrata e il suo sviluppo storico «in ascolto dello Spirito», in: Conferenza Italiana Superiori Maggiori (red.), Consacrati da Dio, dono alla Chiesa e al mondo. Approfondimenti sull'Esortazione «Vita Consecrata», Roma 1997, 97-114.

Concilio Vaticano II, Costituzione dogmatica sulla Chiesa Lumen gentium, Roma 1964. 
Concilio Vaticano II, Decreto sul rinnovamento della vita religiosa Perfectae caritatis, Roma 1965.

Conferenza Italiana Superiori Maggiori (red.), Consacrati da Dio, dono alla Chiesa e al mondo. Approfondimenti sull'Esortazione "Vita Consecrata», Roma 1997.

Congregazione dei Sacerdoti del Sacro Cuore di Gesù (Dehoniani), Costituzioni, Milano-Napoli 1987.

Crippa L., Opzione preferenziale per i poveri, in: Dizionario Teologico della Vita Consacrata, Milano 1994, 1174-1187.

De Fiores S., Goffi T. (red.), Nuovo dizionario di spiritualità, Cinisello Balsamo (MI) 1999.

Dizionario degli istituti di perfezione, VIII, Roma 1988.

Dizionario enciclopedico di spiritualità, Roma 1995.

Dizionario Teologico della Vita Consacrata, Milano 1994.

Estrada Díaz J. A., Imitazione di Gesù Cristo, in: Dizionario Teologico della Vita Consacrata, Milano 1994, 835-849.

Galot J., Consacrazione battesimale e consacrazione religiosa, „Vita Consacrata” 12(14) (1978), 590-600.

Galot J., Cristo fondatore della vita consacrata, „Vita Consacrata” 4(27) (1991), 306-315.

Galot J., La Trasfigurazione, „Vita Consacrata” 3-4(32) (1996), 351-362.

Gambari E., Manuale della vita religiosa, Roma 1970, 155-158.

Gesteira Garza M., Eucaristia, in: Dizionario Teologico della Vita Consacrata, Milano 1994, 695-721.

Ghirlanda G. F., L'esortazione apostolica Vita Consecrata continua a provocare i consacrati, „Vita Consacrata” 2(34) (1998), 140-155.

Giovanni Paolo II, Esortazione apostolica Redemptionis donum, Roma 1984.

Giovanni Paolo II, Esortazione apostolica Vita consecrata, Roma 1996.

Gonzáles Silva S., Consacrati e inviati, in: Vita Consecrata. Una prima lettura teologica, Milano 1996, 107-130. 
Goya B., Formazione integrale alla vita consacrata alla luce della esortazione post-sinodale, Bologna 1997.

Gribomont J., Professione e battesimo, in: Dizionario degli istituti di perfezione, VII, Roma 1983, 890-892.

Guccini L., Religiosi e laici insieme verso il domani, in: Una comunità per domani. Prospettive della vita religiosa apostolica, Bologna 2000, 57-79.

IX Sinodo dei Vescovi, La vita consacrata. Proposte, Roma 1994, in: Enchiridion Vaticanum, T. 14, Bologna 1997, 1565-1680.

La vita consacrata. Un carisma da riscoprire nella Chiesa comunione-missione, Padova 1994.

Leclercq G., Professione religiosa secondo battesimo, in: „Vita Religiosa” 1 (1967), 3-8.

Maggioni B., Il fondamento evangelico della vita consacrata, in: Vita consacrata un dono del Signore alla sua Chiesa, Leumann (Torino) 1994, 93-128.

Mantovani M., Un «tempo di gioia»: la formazione iniziale alla vita consacrata, in: P. Vanzan, F. Volpi (red.), Il giubileo e la vita consacrata. Provocazioni e risposte, Roma 2001, 149-161.

Matura T., Il radicalismo evangelico, Roma 1981.

Mistrorigo A., Consigli evangelici, in: A. Mistrorigo, Guida alfabetica alla vita spirituale, Casale Monferrato 1998, 82-84.

Palmés de Genover C., Battesimo, in: Dizionario Teologico della Vita Consacrata, Milano 1994, 119-133.

Palumbieri S., "Gioia» e vita consacrata, in: P. Vanzan, F. Volpi (red.), Il giubileo e la vita consacrata. Provocazioni e risposte, Roma 2001, 111-124.

Pigna A., Fondamenti teologici della vita consacrata, „Rivista di Vita Spirituale” 51 (1997), 72-97.

Pigna A., I contenuti, „Vita Consacrata” 3-4(32) (1996), 291-311.

Pigna A., Il dono dei consigli evangelici, „Rivista di Vita Spirituale” 54 (2000), 173-192.

Pigna A., Risposta della esortazione alle questioni poste dal sinodo, in: Conferenza Italiana Superiori Maggiori (red.), Consacrati da Dio, dono alla Chiesa e al mondo. Approfondimenti sull'Esortazione "Vita Consecrata», Roma 1997, 183-221. 
Poli G. F., La teologia della vita consacrata dal Concilio ad oggi, in: La vita consacrata. Un carisma da riscoprire nella Chiesa comunione-missione, Padova 1994, 81-112.

Proietti B., Sequela Christi e imitazione, in: Dizionario degli istituti di perfezione, VIII, Roma 1988, 1287-1314.

Pussino G. L., Laici e persone consacrate: quali rapporti?, in: Conferenza Italiana Superiori Maggiori (red.), Consacrati da Dio, dono alla Chiesa e al mondo. Approfondimenti sull'Esortazione «Vita Consecrata», Roma 1997, 337-356.

Ruffini E., Eucaristia, in: S. De Fiores, T. Goffi (red.), Nuovo dizionario di spiritualità, Cinisello Balsamo (MI) 1999, 601-622.

Sanna I., Mistero pasquale, in: S. De Fiores, T. Goffi (red.), Nuovo dizionario di spiritualità, Cinisello Balsamo (MI) 1999, 971-984.

Sartorio U., La testimonianza carismatica dei consacrati nella Chiesa e di fronte al mondo, in: La vita consacrata. Un carisma da riscoprire nella Chiesa comunione-missione, Padova 1994, 205-264.

Savagnone G., Il sale e la luce. Il contributo specifico della vita consacrata al progetto culturale, in: Conferenza Italiana Superiori Maggiori (red.), Consacrati da Dio, dono alla Chiesa e al mondo. Approfondimenti sull'Esortazione «Vita Consecrata», Roma 1997, 315-336.

Secondin B., Guardando al futuro, in: Vita Consecrata. Una prima lettura teologica, Milano 1996, 171-183.

Secondin B., Il profumo di Betania. La vita consacrata come mistica, profezia, terapia, Bologna 1997.

Una comunità per domani. Prospettive della vita religiosa apostolica, Bologna 2000.

Valentini G., Lopzione preferenziale per i poveri è di tutti, ma soprattutto dei consacrati, in: P. Vanzan, F. Volpi (red.), Il giubileo e la vita consacrata. Provocazioni e risposte, Roma 2001, 165-173.

Vanzan P., Volpi F. (red.), Il giubileo e la vita consacrata. Provocazioni e risposte, Roma 2001.

Vita consacrata un dono del Signore alla sua Chiesa, Leumann (Torino) 1994.

Vita Consecrata. Studi e riflessioni, Roma 1996.

Vita Consecrata. Una prima lettura teologica, Àncora, Milano 1996. 
Zambotti F., Le nuove povertà interpellano la vita consacrata, in: P. Vanzan, F. Volpi (red.), Il giubileo e la vita consacrata. Provocazioni e risposte, Roma 2001, 175-180.

Ks. Leszek Poleszak - sercanin, doktor teologii, rektor Wyższego Seminarium Misyjnego Księży Sercanów w Stadnikach, członek Polskiego Stowarzyszenia Teologów Duchowości. W pracy naukowej zajmuje się teologią Najświętszego Serca Jezusowego oraz duchowością. 